\title{
X-ray variability of Capella ${ }^{\star}$
}

\author{
A. J. J. Raassen ${ }^{1,2}$ and J. S. Kaastra ${ }^{1}$ \\ 1 SRON Netherlands Institute for Space Research, Sorbonnelaan 2, 3584 CA Utrecht, The Netherlands \\ e-mail: a.j.j.raassen@sron.nl \\ 2 Astronomical Institute Anton Pannekoek, Kruislaan 403, 1098 SJ Amsterdam, The Netherlands
}

Received 23 May 2006 / Accepted 21 September 2006

\begin{abstract}
We discuss 12 individual observations of stable calibration source Capella over the last five years. The light curves and spectra, derived from $L E T G S$ on board CHANDRA are investigated. The spectra cover the wavelength range 1-175 $\AA$. Nine of the twelve observations show comparable count rates, while the three most recent observations between March 2005 and April 2006 show a 40-50\% higher count rate. No individual flares were recognised. Line flux ratios between different observations show that the variations are related to the hotter plasma. Variations in line fluxes of highly ionised iron lines show a strong resemblance with earlier line flux variations in the years 1992-1995. A time interval of 10.7(.2) year is determined between two maxima in line fluxes. Emission measure modellings to the total spectra have been made by means of SPEX in combination with MEKAL. Observations with higher count rates have a higher emission measure at higher temperature.
\end{abstract}

Key words. stars: individual: Capella - stars: coronae

\section{Introduction}

The first line rich $\mathrm{X}$-ray source observed by CHANDRA and XMM-Newton was Capella, a G1III+G8III giant binary system. The spectrum of this source shows an overwhelming amount of emission lines in the range from 0.07 to $12 \mathrm{keV}$, i.e. from 1 to $175 \AA$. Therefore Capella serves as source for calibrating and testing (resolving power, response matrix, line spread function, etc) the instruments aboard the new X-ray space observatories (Canizares et al. 2005). It is described in the first light papers on high resolution (grating) spectroscopy of both satellites (Canizares et al. 2000 (HETG); Brinkman et al. 2000 (LETG) and Audard et al. 2001 (RGS)). Thanks to the many resolved line features, the spectra offer the possibility to investigate the coronal plasma into detail. These investigations have resulted in a number of papers on temperatures, emission measures and abundance determinations of the corona of Capella, based on $\mathrm{X}$-ray spectra from CHANDRA and XMM-Newton (e.g. Behar et al. 2001; Mewe et al. 2001; Ness et al. 2001; Desai et al. 2005). Over these years Capella has shown to be stable without any evidence of flaring. However, in the past some variation (Linsky et al. 1998; Johnson et al. 2002; Brickhouse et al. 2000) was noticed, but also without flaring.

Before the CHANDRA and XMM-Newton era Linsky et al. (1998) have investigated the Capella spectrum with EUVE over the years 1992 to 1995 . They focussed on the fluxes of a number of Fe lines at 128.73, 97.88, 102.22, 142.27, 108.37, 93.92 $\AA$ and determined the emission measures from these Fe line fluxes at different times. Based on the variability of the volume emission measures, they conclude to variability in the coronal emission measure at the hotter temperatures.

Linsky et al. (1998) and Johnson et al. (2002) investigated the Fe XXI line at $1354 \AA$ using the Hubble Space

$\star$ Table 1 and Fig. 2 are only available in electronic form at http://www. aanda.org
Telescope/Space Telescope Imaging Spectrograph in 1995 and 1999, respectively. Comparing both observations Johnson et al. (2002) notice a decline by a fairly large factor of the emission of this line related to the G8III star in the binary system. Ishibashi et al. (2006) assume that the G8III star is the strongest X-ray source, but that the G1III star significantly contributes to the hotter emission at $T \gtrsim 10^{7} \mathrm{~K}$.

In this paper we investigate the high resolution X-ray spectra of Capella observed by LETGS on board CHANDRA over the last six years. We include in this paper the investigations of light curves, the investigations of individual line fluxes and line flux ratios, as well as overall spectral fittings to the total spectra by means of SPEX.

In Sect. 2 the observations are discussed and the log of the observations is given. In Sect. 3 we describe the light curves and their fluctuations. Section 4 shows the individual line fluxes and their behaviour over the years, while in Sect. 5 a DEM-modelling to the total spectra is discussed.

\section{Observations}

Twenty one spectra of Capella were obtained with $L E T G$ on board CHANDRA over a period of several years, from the launch of CHANDRA in 1999 till now, as part of the calibration program. Here we focus on the twelve LETG-spectra that were detected with $H R C$ - $S$ and that have exposure times $\gtrsim 20 \mathrm{ks}$.

We used the archival data (including the CIAO pipelining) over the years 1999-2006. Light curves and spectra were extracted for all (twelve) observations of this calibration source. The log of the observations is shown in Table 1. Eleven of the observations have durations between 25 and $35 \mathrm{ks}$, and one long observation in November 1999 (Obs ID=1248) has a duration of $85 \mathrm{ks}$. Seven observations are on-axis, while five are off-axis. The offset in Table 1 is given in arcmin. A minus sign indicates a lower chipy coordinate and a lowering of the upper wavelength 


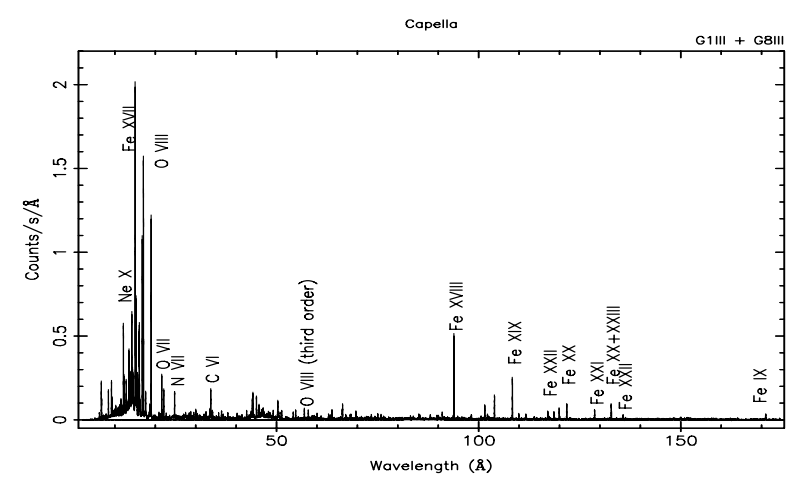

Fig. 1. The combined + and - order spectrum of Capella of Obs ID $=1248$ obtained with $L E T G S$ on board CHANDRA.

of the +1 order by $3.36 \AA$ per arcmin. Telescope vignetting influences results for $E \gtrsim 1.5 \mathrm{keV}$, and only slightly for $E \lesssim 1.5 \mathrm{keV}$. For an offset $\lesssim 4$ arcmin errors from not accounting for vignetting are smaller than $4 \%$.

The source spectra were extracted from a box in crossdispersion of size $h=\max (1.02,0.027|\lambda|)$. Here $h$ is the half width, given in arcsec. The background was extracted from boxes at both sides of the source spectrum with cross-dispersion angles between 10 and 39.5 arcsec.

Thanks to the high flux of the source all observations show a line rich spectrum with high signal to noise $(\mathrm{S} / \mathrm{N})$ ratio (see Fig. 1). This figure is the combined + and - order spectrum of observation $\mathrm{Obs}$ ID=1248 with an exposure time of $85 \mathrm{ks}$. A number of ions are indicated in this figure. Figure 1 shows a variety of ions: $\mathrm{H}-$ and He-like ions of carbon, nitrogen, oxygen, neon, magnesium, and silicon together with many ionisation stages of iron (Fe IX and Fe XVII to Fe XXIII). These types of spectra are very well suited to investigate coronal plasma properties.

\section{Light curves}

Based on the (CIAO pipelined) archival data, light curves have been obtained extracted from an area around the 0th order image, covering $90 \%$ of the counts. The count rates are not sensitive to off-axis positioning as long as the off axis is below $4 \operatorname{arcmin}^{1}$. The size of the time bins of our light curve is chosen to be $1000 \mathrm{~s}$, to avoid influences by dithering. The light curves of two observations $(\mathrm{Obs} \mathrm{ID}=1248$ and $\mathrm{Obs} \mathrm{ID}=6165)$ are shown in Fig. 2. All light curves are flat. During none of the observations individual flares are recognised. The horizontal lines in this figure indicate the average count rate for each of the light curves.

The dates and count rates of the light curves of all observations ordered by time are collected in Table 2 . The table contains observation identification number, the dates of observation, the Julian Day-values, the count rates and the standard deviations. The count rates are determined by fitting a constant through the light curve data.

For all, except the four most recent observations the count rates are within 5\%, i.e., between 1.898 and 2.121. The observation Obs ID $=5041$ might give already the first indication for the rise to the higher count rate values of the observations Obs ID $=5956$, Obs ID=6165, and Obs ID=6472 obtained from March 2005 till April 2006. The count rates of these observations

\footnotetext{
1 http://cxc.harvard.edu/proposer/POG/pog_ps.html page 132 .
}

Table 2. Average count rates of Capella, based on the LETGS observations over a period of years from the launch of CHANDRA in 1999 to now, ordered by time. The count rates are obtained from averaging over the time bins of $1000 \mathrm{~s}$.

\begin{tabular}{llllr}
\hline \hline Obs ID & Date of observation & JD & Counts/s & St.dev. \\
\hline 62435 & $1999-09-06$ T05 & 2451427.7 & 1.969 & 0.066 \\
1420 & $1999-10-30 T 00$ & 2451481.5 & 2.089 & 0.051 \\
1248 & $1999-11-10 T 01$ & 2451492.5 & 2.121 & 0.051 \\
0058 & $2000-03-08 T 12$ & 2451612.0 & 2.074 & 0.052 \\
1009 & $2001-02-14 T 15$ & 2451955.1 & 1.985 & 0.046 \\
2582 & $2002-10-05 T 04$ & 2452552.7 & 2.114 & 0.049 \\
3479 & $2002-10-06$ T15 & 2452554.1 & 2.020 & 0.048 \\
3675 & $2003-09-28 T 08$ & 2452910.8 & 1.898 & 0.053 \\
5041 & $2004-09-11 T 11$ & 2453260.0 & 2.193 & 0.043 \\
5956 & $2005-03-31 T 12$ & 2453461.0 & 2.768 & 0.054 \\
6165 & $2005-10-02 T 08$ & 2453645.7 & 3.048 & 0.053 \\
6472 & $2006-04-24 T 04$ & 2453849.5 & 2.592 & 0.048 \\
\hline
\end{tabular}

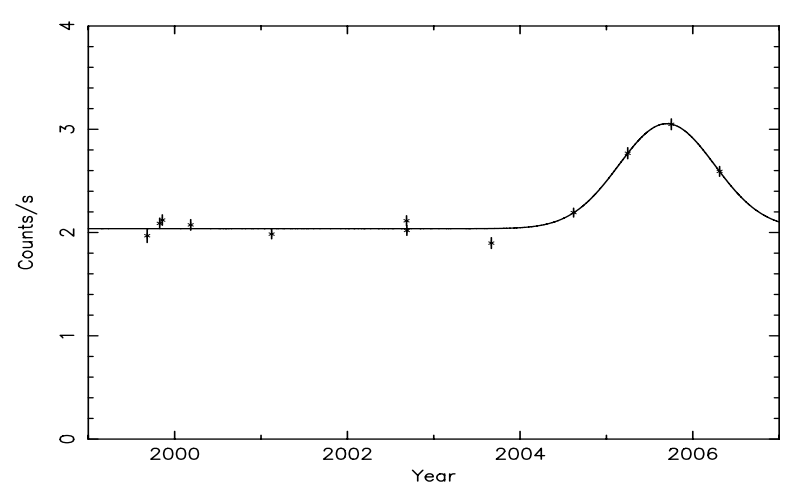

Fig. 3. The average count rates (counts/s) of the Capella, based on the LETGS observations over a period of years from the launch of CHANDRA in 1999 till now.

are about $40-50 \%$ higher than the count rates of the other observations. Although a variability in count rates between the observations $\mathrm{Obs} I D=5956$, Obs ID $=6165$, and $\mathrm{Obs} \mathrm{ID}=6472$, and the other observations is found no short duration flares are noticed in any of the light curves. The average count rate versus the time is shown in Fig. 3. The error bars on the data points are the standard deviation values given in Table 2. This figure shows the steep increase starting from September 2004. By fitting a Gaussian profile in combination with a constant, the peak was determined to be 12 September 2005 with an error of 11 days. The half-width at half maximum of the Gaussian is 203(12) days. The average count rate for the flat part (end of 1999 till the end of 2003) is 2.03(.07) counts/s, while the peak value is $1.02(.05)$ counts/s higher. This is an increase of $50 \%$ in count rate.

In Sect. 4 this increase will be discussed in relation to the variability of individual line fluxes.

\section{Individual lines}

\subsection{Line flux ratios versus temperature}

We have measured the individual line fluxes of a number of prominent lines in the LETGS spectrum of Capella. An extended investigation to identify most features in the LETG spectrum of Capella was done by Mewe et al. (2001), but is outside the scope of this paper. Here we focus on variability. The line flux measurement was done by folding a Gaussian profile through the response matrix and by fitting to the individual line features over 
Table 3. Line fluxes of Obs $\mathrm{ID}=1248$ and 5956 in units of $10^{-13}$ ergs $\mathrm{cm}^{-2} \mathrm{~s}^{-1}$ as measured at Earth. The last column gives the line flux ratios, as used in Fig. 4. The values within brackets are the $1 \sigma$ errors.

\begin{tabular}{lrrrrr}
\hline \hline ion & $\lambda$ & $T_{\text {opt }}$ & flux & flux & ratio \\
& & & 1248 & 5956 & \\
\hline Fe IX & 171.075 & 5.86 & $3.56(.42)$ & $4.07(.72)$ & $1.13(.24)$ \\
C VI & 33.736 & 6.13 & $4.78(.26)$ & $5.82(.52)$ & $1.19(.12)$ \\
N VI & 28.787 & 6.17 & $1.18(.14)$ & $1.07(.26)$ & $0.90(.24)$ \\
N VI & 29.520 & 6.17 & $1.06(.14)$ & $1.16(.28)$ & $1.10(.31)$ \\
N VII & 24.781 & 6.32 & $5.23(.27)$ & $6.37(.52)$ & $1.22(.12)$ \\
O VII & 21.602 & 6.33 & $8.16(.35)$ & $8.13(.62)$ & $1.00(.09)$ \\
O VII & 22.101 & 6.33 & $5.62(.30)$ & $6.31(.55)$ & $1.12(.11)$ \\
Fe XVI & 50.350 & 6.45 & $2.28(.14)$ & $2.59(.26)$ & $1.14(.13)$ \\
Fe XVI & 50.555 & 6.45 & $0.83(.11)$ & $1.05(.20)$ & $1.28(.30)$ \\
Fe XVI & 66.263 & 6.46 & $1.30(.14)$ & $1.37(.34)$ & $1.05(.28)$ \\
Fe XVI & 66.368 & 6.46 & $1.84(.16)$ & $2.14(.38)$ & $1.16(.23)$ \\
O VIII & 18.969 & 6.48 & $27.18(.53)$ & $31.94(1.0)$ & $1.18(.04)$ \\
Fe XVII & 15.013 & 6.72 & $47.40(1.0)$ & $55.22(1.5)$ & $1.16(.04)$ \\
Fe XVII & 16.775 & 6.72 & $26.80(.55)$ & $33.23(1.8)$ & $1.24(.05)$ \\
Fe XVII & 17.051 & 6.72 & $37.31(1.1)$ & $44.97(2.8)$ & $1.21(.08)$ \\
Fe XVII & 17.101 & 6.72 & $30.91(1.1)$ & $38.98(2.7)$ & $1.26(.10)$ \\
Ne X & 12.134 & 6.77 & $18.73(.58)$ & $22.55(1.2)$ & $1.20(.07)$ \\
Fe XVIII & 93.923 & 6.80 & $8.50(.23)$ & $10.64(.44)$ & $1.25(.06)$ \\
Fe XVIII & 103.937 & 6.80 & $3.11(.15)$ & $3.62(.30)$ & $1.16(.11)$ \\
Fe XIX & 108.37 & 6.89 & $5.51(.20)$ & $7.10(.38)$ & $1.29(.08)$ \\
Fe XX & 118.66 & 6.95 & $1.08(.11)$ & $1.80(.29)$ & $1.67(.32)$ \\
Fe XX & 121.83 & 6.95 & $2.20(.16)$ & $3.46(.31)$ & $1.57(.18)$ \\
Mg XII & 8.421 & 7.01 & $5.01(.33)$ & $7.80(.75)$ & $1.55(.18)$ \\
Fe XXI & 128.73 & 7.02 & $2.19(.18)$ & $4.13(.42)$ & $1.89(.24)$ \\
Fe XXII & 117.17 & 7.08 & $1.33(.13)$ & $2.54(.24)$ & $1.91(.26)$ \\
Si XIV & 6.182 & 7.21 & $1.87(.24)$ & $3.73(.56)$ & $2.00(.40)$ \\
\hline & & & & &
\end{tabular}

a limited wavelength range. A power law distribution was added to adjust for the continuum.

The obtained fluxes were used to calculate the line flux ratios between the lines of observations $\mathrm{Obs} I D=1248$, the on-axis observation with the longest exposure time, and Obs ID=5956. The latter being one of the three observations with higher zeroth order count rate, but just like Obs ID $=1248$ on-axis. For the off-axis observations telescope vignetting might influence the fluxes of the Si XIV and Mg XII lines at 6.182 and $8.421 \AA$, respectively. There may also be a small uncertainty of the crossdispersion factor for off-axis observations. The line fluxes are collected in Table 3 in order of increasing optimal line formation temperature $\left(T_{\mathrm{opt}}\right)$, given on a $\log$ scale. This is the temperature of the peak of the emissivity distribution. The underlying calculations of the ionisation balance are from Arnaud \& Rothenflug (1985) and Arnaud \& Raymond (1992). The line flux ratios versus the optimal line formation temperature are plotted in Fig. 4. The errors on the temperature are arbitrarily chosen to be 0.1 dex.

The figure shows that the line flux ratios are constant for values of $\log T$ below 6.8 . In this temperature domain the line fluxes of observation Obs ID $=5956$ are a factor of 1.16(.09) higher than for observation $\mathrm{Obs} \mathrm{ID}=1248$. Above $\log T=6.8$ the line flux ratio 5956/1248 increases to about a factor of 2 . This indicates that no overall increase of the emission measure (by a constant factor) is present, but that the increase of the emission measure depends on the temperature. It is higher for the hottest temperature component.

This is in agreement with the conclusions of Linsky et al. (1998) and Johnson et al. (2002). They investigated the Fe XXI line at $1354 \AA$ observed by HST. Thanks to the more

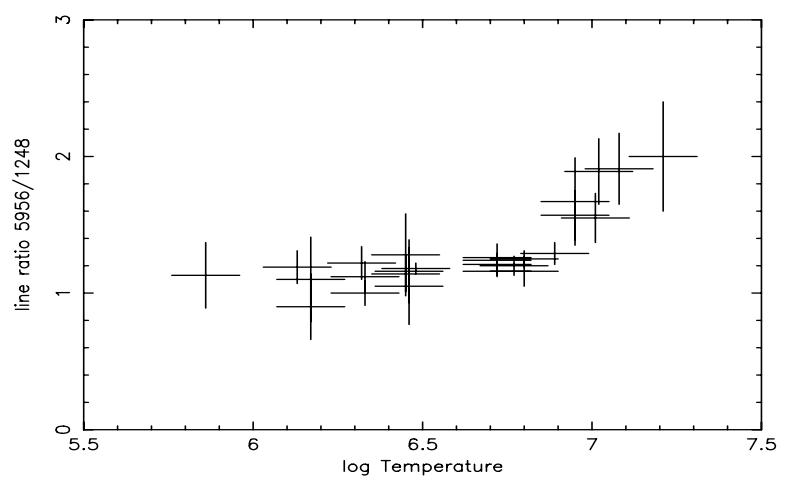

Fig. 4. The line flux ratio between observation Obs ID=5956 and Obs ID=1248 versus the optimal formation temperature.

dispersive wavelength range of the Fe XXI line at $1354 \AA$ Johnson et al. (2002) could assign the variability in emission to the G8III component of the binary system, the star with the hotter coronal plasma. Their conclusion was based on a comparison of their data with those from the earlier observations by Linsky et al. (1998). Ishibashi et al. (2006) assume that the G8III star is the strongest X-ray source, but that the G1III star significantly contributes to the hotter emission at $T \gtrsim 10^{7} \mathrm{~K}$.

\subsection{Line fluxes versus time}

Apart from a comparison of the line flux ratios of a large number of lines of the observations Obs ID=1248 and Obs ID=5956 versus the temperature (this work) we have measured for all twelve LETGS observations the fluxes of the the lines at $93.92 \AA$ (Fe XVIII), $108.37 \AA$ (Fe XIX), and $128.73 \AA$ (Fe XXI). They were observed by Linsky et al. (1998) and Brickhouse et al. (2000) and were indicated to be unblended. The results of the series using EUVE (Linsky et al. 1998; Brickhouse et al. 2000) and of this work are collected in Table 4 . The fluxes are given in units of $10^{-13} \mathrm{erg} \mathrm{cm}^{-2} \mathrm{~s}^{-1}$.

Although calibration differences between EUVE and LETGS can not be ruled out, the fluxes obtained by EUVE (Linsky et al. 1998; Brickhouse et al. 2000) are close to the values given in this paper and they are variable over time. According to our conclusions from Fig. 4 the fluxes of the Fe XXI line at $128.73 \AA$ are most sensitive for variations, due to the high formation temperature. This way we may study the behaviour of the line fluxes versus the time. The behaviour of the flux of the line at $128.73 \AA$ versus the time is given in Fig. 5. This figure covers a range of about 15 years. However, there are also severe gaps in observations between March 1996 and September 1999 and between March 1994 and December 1995. Figure 5 shows two clear peaks. To determine the peak values the peaks were fitted with a Gaussian. Due to the absence of EUVE observations between March 1994 and December 1995 (the time of maximum) the peak profiles are forced to have the same shape, by coupling the peak value and the half-width at half maximum. The date of our peak, determined this way, is 16 September 2005 (19 days), while the half-width at half maximum is 215 (19) days. The time difference between the two peaks is 10.7(.2) years. These results are in good agreement with predictions by Raassen \& Kaastra (2006), made before the observation in April 2006. 
Table 4. Line fluxes for the Fe XXI line at $128.73 \AA$, the Fe XIX line at 108.37 $\AA$, and the Fe XVIII line at $93.92 \AA$ in Capella. The fluxes indicated by EUVE are from Linsky et al. (1998) and from Brickhouse et al. (2000), while the others are from this work. The fluxes are corrected for interstellar absorption $\left(N_{\mathrm{H}}=1.8 \times 10^{18} \mathrm{~cm}^{-2}\right)$.

\begin{tabular}{lllll}
\hline \hline Obs ID & \multicolumn{1}{l}{ Date } & \multicolumn{3}{c}{ flux $^{a}$} \\
& & $128.73 \AA$ & $108.37 \AA$ & $93.92 \AA$ \\
\hline EUVE $^{b}$ & $1992-12-11$ & $2.2(0.3)$ & $4.4(0.3)$ & $7.3(0.4)$ \\
EUVE $^{b}$ & $1993-12-23$ & $2.5(0.3)$ & $3.5(0.3)$ & $5.3(0.4)$ \\
EUVE $^{b}$ & $1994-02-15$ & $4.8(0.5)$ & $6.1(0.4)$ & $8.4(0.6)$ \\
EUVE $^{b}$ & $1994-02-26$ & $4.6(0.4)$ & $6.8(0.4)$ & $8.4(0.6)$ \\
EUVE $^{b}$ & $1995-12-02$ & $3.7(0.4)$ & $7.8(0.4)$ & $10.1(0.6)$ \\
EUVE $^{c}$ & $1996-03-05$ & $2.6(0.2)$ & $5.5(0.2)$ & $10.2(0.3)$ \\
62435 & $1999-09-06$ & $2.1(0.3)$ & $5.5(0.3)$ & $8.5(0.4)$ \\
1420 & $1999-10-30$ & $2.6(0.4)$ & $5.4(0.3)$ & $9.2(0.4)$ \\
1248 & $1999-11-10$ & $2.4(0.2)$ & $5.9(0.2)$ & $8.9(0.2)$ \\
0058 & $2000-03-08$ & $2.4(0.3)$ & $5.4(0.3)$ & $8.2(0.4)$ \\
1009 & $2001-02-14$ & $2.1(0.3)$ & $4.6(0.3)$ & $8.4(0.4)$ \\
2582 & $2002-10-05$ & $2.0(0.4)$ & $5.6(0.4)$ & $9.1(0.4)$ \\
3479 & $2002-10-06$ & $2.2(0.5)$ & $5.2(0.4)$ & $8.6(0.5)$ \\
3675 & $2003-09-28$ & $1.7(0.3)$ & $4.7(0.3)$ & $7.9(0.4)$ \\
5041 & $2004-09-11$ & $3.0(0.4)$ & $6.6(0.4)$ & $9.3(0.4)$ \\
5956 & $2005-03-31$ & $4.6(0.4)$ & $7.6(0.4)$ & $11.1(0.4)$ \\
6165 & $2005-10-02$ & $5.4(0.5)$ & $9.2(0.4)$ & $12.5(0.5)$ \\
6472 & $2006-04-24$ & $4.2(0.4)$ & $7.7(0.4)$ & $10.5(0.4)$ \\
\hline
\end{tabular}

${ }^{a}$ In units of $10^{-13} \mathrm{erg} \mathrm{cm}^{-2} \mathrm{~s}^{-1}$.

${ }^{b}$ From Linsky et al. (1998).

${ }^{c}$ From Brickhouse et al. (2000).

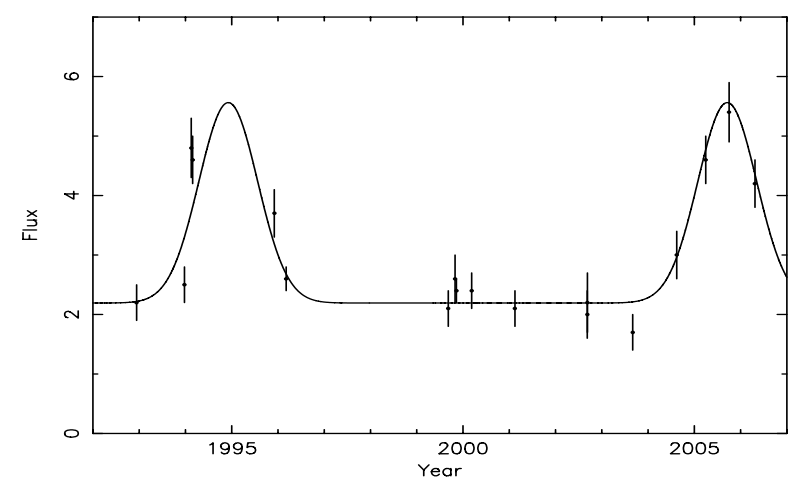

Fig. 5. The line flux of Fe XXI line $\lambda=128.73$ versus time. Over the years 1992 to 1995 we use the data from $E U V E$ (Linsky et al. 1998), for one data point in 1996 from EUVE (Brickhouse et al. 2000) and over the years 1999 to 2005 we use the data from LETGS aboard CHANDRA (this work). The fluxes are in units of $10^{-13} \mathrm{erg} \mathrm{cm}^{-2} \mathrm{~s}^{-1}$.

\section{DEM-modelling}

To have some other confirmation for our conclusion that the increase in emission is related to the hotter plasma (see Fig. 4) we analyze the total LETGS spectra of Obs ID=1248 and Obs ID=6165 by means of a differential emission measure (DEM) modelling, using SPEX (Kaastra et al. 1996a) in combination with MEKAL (Mewe et al. 1985, 1995; Kaastra et al. 1996b). MEKAL calculates a continuum and models more than 5400 spectral lines, and is available on the $\mathrm{wWW}^{2}$. The applied model is a Collisional Ionisation Equilibrium (CIE) model for optically thin plasma. The ionisation equilibrium is based on calculations by Arnaud \& Rothenflug (1985) and Arnaud \& Raymond (1992). In an earlier investigation of the temperature structure of the corona of Capella (Audard et al. 2001) a three

\footnotetext{
${ }^{2}$ http://www.sron.nl/divisions/hea/spex/
}

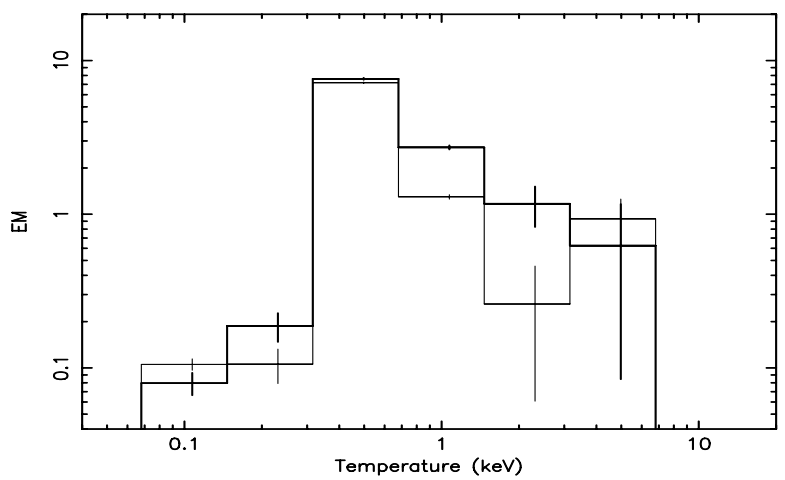

Fig. 6. DEM modelling to two spectra of Capella. The thin line corresponds to $\mathrm{Obs} \mathrm{ID}=1248$ and the bold line to $\mathrm{Obs} \mathrm{ID}=6165$. $E M$ is given in units of $10^{52} \mathrm{~cm}^{-3}$.

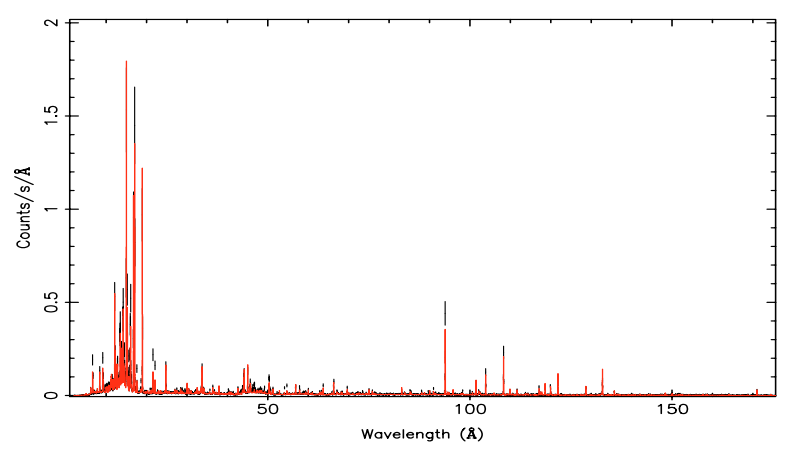

Fig. 7. Modelled and observed spectrum of observation $\mathrm{Obs} I D=1248$. The solid line (red in the electronic version) is the model.

temperature model was applied to RGS spectra obtained March 2000 , close to the dates of our observations Obs ID=1248 and Obs ID $=0058$.

For our differential emission measure (DEM) modelling we use the module "regularisation" (e.g., Kaastra et al. 1996b) in SPEX. This module uses a matrix inversion with an additional constraint that the second derivative of the solution is as smooth as possible. The emission measure is defined as $E M=n_{\mathrm{e}} n_{\mathrm{H}} V$ in which $n_{\mathrm{H}}=0.85 \times n_{\mathrm{e}}$. The model spectrum (including the 5400 lines of MEKAL) is fitted to the total spectrum, line features and continuum, minimising the $\chi^{2}$ value. A wavelength correction (a multiplication factor of $-4.6 \times 10^{-4}$ for Obs ID $=1248$ and of $-1.05 \times 10^{-3}$ for Obs ID=6165) was added to correct for wavelength deviations. Abundances by Audard et al. (2001) based on XMM-Newton and by Argiroffi et al. based on LETGS agree except for the value of Si. It is clear from Fig. 1 in Audard et al. (2001) that the value of Argiroffi et al. (2003) is better. We applied these abundances for the DEM-modelling.

The result for the $E M$ (per logarithmic temperature bin) is shown in Fig. 6. The $E M$ is given in units of $10^{52} \mathrm{~cm}^{-3}$. The bold line is related to the DEM of the spectrum of observation Obs ID $=6165$. The thinner line belongs to observation $\mathrm{Obs} \mathrm{ID}=1248$. The summed $E M$ of the observation Obs ID $=1248$ is $9.8(0.4) \times 10^{52} \mathrm{~cm}^{-3}$, close to the value $9.60(0.13) \times 10^{52} \mathrm{~cm}^{-3}$ determined by Audard et al. (2001) and the $10.8(0.7) \times 10^{52} \mathrm{~cm}^{-3}$ by Argiroffi et al. (2003). From this figure it is clear that the observation of Obs ID=6165 has a significantly higher emission than observation $\mathrm{Obs}$ ID=1248 around $1 \mathrm{keV}$.

Figure 7 shows the observed and modelled spectrum of observation Obs ID $=1248$, based on the DEM-modelling. 
Table 5. Calculated and measured line fluxes of Obs ID=1248 and 6165 in units of $10^{-13} \mathrm{erg} \mathrm{cm}^{-2} \mathrm{~s}^{-1}$.

\begin{tabular}{lrrrrrrr}
\hline \hline ion & \multicolumn{3}{c}{$\lambda$} & \multicolumn{3}{c}{ flux 1248} & \multicolumn{3}{c}{ flux 6165} & \multicolumn{3}{c}{ ratio } \\
& \multicolumn{3}{c}{ calc. } & meas. calc. & meas. calc. meas. \\
\hline Fe IX & 171.075 & 4.4 & 3.6 & 2.5 & 2.3 & 0.6 & 0.6 \\
C VI & 33.736 & 5.2 & 4.8 & 5.3 & 4.7 & 1.0 & 1.0 \\
N VII & 24.781 & 5.1 & 5.2 & 6.3 & 5.3 & 1.2 & 1.0 \\
O VII & 21.602 & 4.6 & 8.2 & 3.9 & 8.7 & 0.9 & 1.1 \\
O VIII & 18.969 & 34.9 & 27.2 & 38.0 & 31.2 & 1.1 & 1.1 \\
Fe XVII & 15.013 & 58.4 & 47.4 & 64.1 & 51.8 & 1.1 & 1.1 \\
Fe XVII & 16.775 & 29.8 & 26.8 & 32.2 & 30.6 & 1.1 & 1.1 \\
Ne X & 12.134 & 22.0 & 18.7 & 25.3 & 25.6 & 1.2 & 1.4 \\
Fe XVIII & 93.923 & 7.8 & 8.5 & 9.0 & 11.9 & 1.2 & 1.4 \\
Fe XIX & 108.37 & 5.6 & 5.5 & 7.6 & 8.6 & 1.4 & 1.6 \\
Fe XX & 121.83 & 3.8 & 2.2 & 6.3 & 4.4 & 1.7 & 2.0 \\
Mg XII & 8.421 & 4.8 & 5.0 & 7.3 & 6.9 & 1.5 & 1.4 \\
Fe XXI & 128.73 & 3.1 & 2.2 & 6.6 & 4.8 & 2.1 & 2.2 \\
Fe XXII & 117.17 & 1.4 & 1.3 & 3.1 & 3.4 & 2.2 & 2.6 \\
Si XIV & 6.182 & 1.6 & 1.9 & 3.1 & 3.2 & 1.9 & 1.7 \\
\hline
\end{tabular}

The calculated and measured fluxes of the observations OBS ID $=1248$ and 6165 are collected in Table 5. Keeping in mind that the theoretical transition probabilities are often off by $25 \%$ the agreement between the calculated and measured fluxes are quite good and reflect the trend given in Fig. 4 .

\subsection{Conclusions}

Twelve spectra of calibration source Capella, observed since 1999 with LETGS aboard CHANDRA, have been studied. They show clear variations in count rates as well as in fluxes of individual highly ionised $\mathrm{Fe}$ lines.

The light curves for all observations are flat (at different count rate level) without the presence of individual flares. However, over years they vary on a scale of $50 \%$. Count rates and line fluxes reached a maximum around 15 September 2005.
The variation in line fluxes depends on the ionisation stages of the ions and the temperature of the peak of the emissivity distribution. The variability is stronger for the hotter coronal plasma.

A model fit through our measured line fluxes of highly ionised Fe lines with measurements by Linsky et al. (1998) and Brickhouse et al. (2000), based on EUVE observation, show two peaks with a time interval of 10.7 (.2) year. To conclude whether it concerns an incidental effect or a periodically returning event regular observations till the year 2017 are necessary.

Acknowledgements. The SRON Netherlands Institute for Space Research is supported financially by NWO.

\section{References}

Argiroffi, C., Maggio, A., \& Peres, G. 2003, A\&A, 404, 1049 Arnaud, M., \& Raymond, R. 1985, ApJ, 398, 394

Arnaud, M., \& Rothenflug, J. 1992, A\&AS, 60, 425

Audard, M., Behar, E., Güdel, et al. 2001, A\&A, 365, L329

Behar, E., Cottom, J., \& Kahn, S. M. 2001, ApJ, 548, 966

Brickhouse, N. S., Dupree, A. K., Edgar, R. J., et al. 2000, ApJ, 530, 387

Brinkman, A. C., Gunsing, C. J. T., Kaastra, J. S., et al. 2000, ApJ, 530, L111

Canizares, C. R., Davis, J. E., Dewey, D., et al. 2005, PASP, 117, 1144

Canizares, C. R., Huenemoerder, D. P., Davis, D. S., et al. 2000, ApJ, 539, L41

Desai, P., Brickhouse, N. S., Drake, J. J., et al. 2005, ApJ, 625, L59

Ishibashi, K., Dewey, D., Huenemoerder, D. P., \& Testa, P. 2006, ApJ, 644, L117

Johnson, O., Drake, J. J., Kashyap, V., et al. 2002, ApJ, 565, L97

Kaastra, J. S., Mewe, R., \& Nieuwenhuijzen, H. 1996a, in UV and X-ray Spectroscopy of Astrophysical and Laboratory Plasmas, ed. K. Yamashita, \& T. Watanabe (Tokyo: Universal Academy Press, Inc.), 411 (SPEX)

Kaastra, J. S., Mewe, R., Liedahl, D. A., et al. 1996b, A\&A, 314, 547

Linsky, J. L., Wood, B. E., Brown, A., \& Osten, R. A. 1998, ApJ, 492, 767

Mewe, R., Gronenschild, E. H. B. M., \& van den Oord, G. H. J. 1985, A\&AS, 62, 197

Mewe, R., Kaastra, J. S., \& Liedahl, D. A. 1995, Legacy, 6, 16 (MEKAL)

Mewe, R., Raassen, A. J. J., Drake, J. J., et al. 2001, A\&A, 368, 888

Ness, J.-U., Mewe, R., Schmitt, J. H. M. M., et al. 2001, A\&A, 367, 282

Raassen, A. J. J., \& Kaastra, J. S. 2006, Workshop on High resolution X-ray spectroscopy: toward XEUS and Con-X, 27-28 March 2006, MSSL, Dorking, UK 
A. J. J. Raassen and J. S. Kaastra: LETGS X-ray spectra of Capella, Online Material $p 1$

\section{Online Material}


A. J. J. Raassen and J. S. Kaastra: LETGS X-ray spectra of Capella, Online Material $p 2$

Table 1. Dates of observation of Capella by means of LETGS.

\begin{tabular}{|c|c|c|c|c|}
\hline Obs ID & Date-obs-start & $\overline{\text { Date-obs-end }}$ & Exposure time(ks) & offset $^{a}$ \\
\hline 62435 & 1999-09-06Т00:26:00 & 1999-09-06Т09:48:00 & 32.71 & -0.13 \\
\hline 1420 & 1999-10-29T22:30:00 & 1999-10-30Т07:27:00 & 30.19 & -0.04 \\
\hline 1248 & 1999-11-09T13:27:00 & 1999-11-10T13:28:00 & 85.23 & 0.00 \\
\hline 0058 & 2000-03-08T06:29:00 & 2000-03-08T16:24:00 & 34.11 & -0.08 \\
\hline 1009 & 2001-02-14T11:40:00 & 2001-02-14T19:26:00 & 26.97 & -0.03 \\
\hline 2582 & 2002-10-04T23:58:00 & 2002-10-05T08:35:00 & 28.83 & -1.50 \\
\hline 3479 & 2002-10-06T10:03:00 & 2002-10-06T19:10:00 & 30.38 & 1.46 \\
\hline 3675 & 2003-09-28T04:23:00 & 2003-09-28T12:21:00 & 27.16 & 0.05 \\
\hline 5041 & 2004-09-11T06:53:00 & 2004-09-11T15:15:00 & 28.88 & 3.03 \\
\hline 5956 & 2005-03-31T08:09:00 & 2005-03-31T17:03:00 & 30.17 & 0.03 \\
\hline 6165 & 2005-10-02Т04:53:00 & 2005-10-02T12:59:00 & 29.17 & -2.97 \\
\hline 6472 & 2006-04-23T23:30:00 & 2006-04-24T07:46:00 & 30.18 & 1.54 \\
\hline
\end{tabular}

${ }^{a}$ given in arcmin.
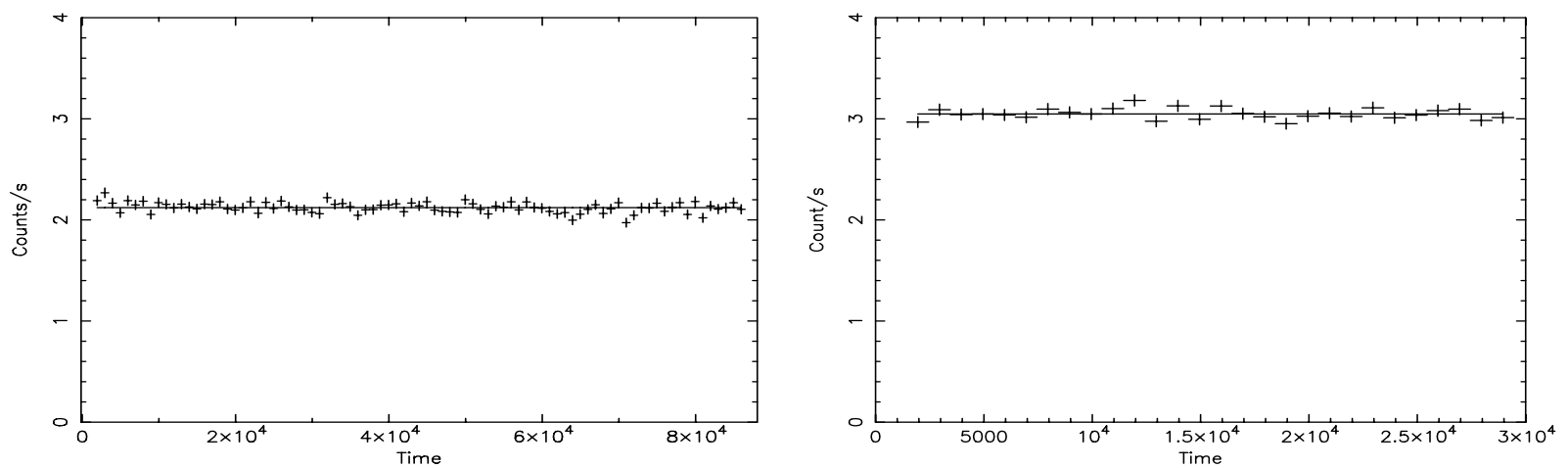

Fig. 2. The light curves of Capella of Obs ID=1248 (left panel) and of Obs ID=6165 (right panel). The time is divided in bins of $1000 \mathrm{~s}$. 